\title{
The Real DFM Radius and Minimum \\ Norm Plant Perturbation for General Control Information Flow Constraints *
}

\author{
Simon Lam and Edward J. Davison* \\ * Systems Control Group, Department of Electrical and Computer \\ Engineering, University of Toronto, Toronto, ON, Canada (E-mail: \\ \{simon,ted\}@control.utoronto.ca).
}

\begin{abstract}
:
The real decentralized fixed mode radius measures how "near" a decentralized LTI system is from having a decentralized fixed mode (DFM) present. In this paper, some properties of the real DFM radius are discussed, a procedure for computing the actual system parametric perturbations that achieve the real DFM radius is presented, and the real DFM radius is extended to deal with structured perturbations and general information flow constraints. A study of applying the radius to determine how to pair system inputs and outputs to obtain a robust decentralized control system structure with respect to parametric perturbations is also presented.
\end{abstract}

\section{INTRODUCTION}

Consider the following decentralized LTI multivariable system with $v$ local control stations described by

$$
\begin{aligned}
\dot{x} & =A x+\sum_{i=1}^{v} B_{i} u_{i} \\
y_{i} & =C_{i} x+\sum_{j=1}^{v} D_{i j} u_{j} \quad(i=1, \ldots, v)
\end{aligned}
$$

where $x \in \mathbb{R}^{n}$ is the state, and $u_{i} \in \mathbb{R}^{m_{i}}$ and $y_{i} \in \mathbb{R}^{r_{i}}$ are respectively the inputs and outputs of the $i$-th control agent $(i=1, \ldots, v)$. The notion of a decentralized fixed mode (DFM) introduced in Wang and Davison (1973) is a generalization of the notion of uncontrollable and unobservable modes of centralized control problems. The importance of DFMs is due to the fact that if system (1) is controlled by a decentralized LTI controller, then the closed-loop system can only be assigned a spectrum that contains the set of DFMs. Consequently, the system can only be decentrally stabilized by a LTI controller if and only if it has no unstable DFMs.

However, simply knowing that a system has no DFMs is often not satisfactory. This is because the system may be very "close" to having one or more DFMs with respect to small parametric perturbations. In other words, a small perturbation in the system's parameters (i.e. $A \rightarrow A+\Delta_{A}$, $B_{i} \rightarrow B_{i}+\Delta_{B_{i}}, C_{i} \rightarrow C_{i}+\Delta_{C_{i}}$, and $D_{i j} \rightarrow D_{i j}+\Delta_{D_{i j}}$ for $i, j=1, \ldots, v)$ may cause the resulting perturbed system to have one or more DFMs. Therefore, a continuous measure of the decentralized assignability of a system's eigenvalues is more desirable than the traditional binary 'yes/no' metric.

Such a measure for the decentralized case, called the DFM radius, was first suggested in Vaz and Davison (1988) and

\footnotetext{
^ This work has been supported by NSERC under grant No. A4396.
}

is based on the approach by Eising (1984) for the centralized case. However, the DFM radius developed in Vaz and Davison (1988) deals only with complex perturbations; i.e. $\Delta_{A}, \Delta_{B_{i}}, \Delta_{C_{i}}$, and $\Delta_{D_{i j}}$, for $i, j=1, \ldots, v$, are complex perturbation matrices. To deal with real perturbations, which is the more realistic case, the DFM radius was recently extended in Lam and Davison (2007), and is based on real perturbation values (see Bernhardsson et al. (1998)).

In this paper, various new results related to the real DFM radius are presented, which are organized as follows. In Section 2, the concepts of real perturbation values, DFMs, and the real DFM radius are briefly reviewed. Section 3 consists of most of the new results related to the real DFM radius. In particular, Section 3.1 presents a procedure for computing the actual system perturbations that achieve the real DFM radius, Section 3.2 discusses some properties of the real DFM radius, and Section 3.3 and 3.4 respectively extends the real DFM radius to deal with structured perturbations and general information flow constraints. Section 4 provides some numerical examples, followed by a study of applying the real DFM radius to determine input/output pairing which is presented in Section 5.

\section{PRELIMINARIES}

The notation used in this paper is standard. The field of real and complex numbers are denoted by $\mathbb{R}$ and $\mathbb{C}$ respectively, and $\mathbb{C}_{+}$denotes the closed right half complex plane. The $i$-th singular value of a matrix $M \in \mathbb{C}^{p \times m}$ is denoted by $\sigma_{i}(M)$, where $\sigma_{1}(M) \geq \sigma_{2}(M) \geq \cdots$. $\bar{M}, M^{T}, M^{*}$, and $M^{+}$denote respectively the complex conjugate, transpose, complex conjugate transpose, and Moore-Penrose pseudoinverse of $M$. $\|M\|$ denotes the spectral norm of $M$ and is equal to $\sigma_{1}(M)$. The real and imaginary components of the matrix $M$ are given by $\Re M$ and $\Im M$ respectively. The eigenvalues of a square matrix $A$ are given by $\operatorname{sp}(A)$. Also, denoting $\mathbb{N}=\{1,2, \ldots\}$, 
then for $n \in \mathbb{N}, \underline{n}$ denotes the set $\{1,2, \ldots, n\}$. Finally, the notation $\left[\frac{A \mid B}{C} \mid \begin{array}{l}D \\ ]_{v}\end{array}\right.$ is used in the rest of the paper to represent a decentralized LTI system of the form (1), where $B:=\left[B_{1} \cdots B_{v}\right], C:=\left[\begin{array}{c}C_{1} \\ \vdots \\ C_{v}\end{array}\right]$, and $D:=\left[\begin{array}{ccc}D_{11} & \cdots & D_{1 v} \\ \vdots & & \vdots \\ D_{v 1} & \cdots & D_{v v}\end{array}\right]$.

\subsection{Real Perturbation Values}

Definition 1. (Real Perturbation Values). Given a matrix $M \in \mathbb{C}^{q \times l}$, the $k$-th real perturbation value of $M, \tau_{k}(M)$, is defined as

$$
\tau_{k}(M):=\inf \left\{\|\Delta\| \mid \Delta \in \mathbb{R}^{q \times l}, \operatorname{rank}(M+\Delta)<k\right\}
$$

where $k \in \mathbb{N}$. $^{1}$

The real perturbation values have the following properties (see Bernhardsson et al. (1995)).

Lemma 2. (Properties of the Real Perturbation Values).
i) $\tau_{k}(\alpha M)=\alpha \tau_{k}(M), \quad \alpha \in \mathbb{R}$
ii) $\tau_{k}(\bar{M})=\tau_{k}(M)$
iii) $\tau_{k}\left(Q_{1} M Q_{2}\right)=\tau_{k}(M), Q_{1}, Q_{2}$ real orthogonal

The $k$-th perturbation value of a matrix $M \in \mathbb{C}^{q \times l}$ can be computed by the following result.

Theorem 3. (Bernhardsson et al. (1998)). Given $M \in \mathbb{C}^{q \times l}$ and $k \in \mathbb{N}$, then

$$
\tau_{k}(M)=\sup _{\gamma \in(0,1]} \sigma_{2 k-1}\left(\left[\begin{array}{cc}
\Re M & -\gamma \Im M \\
\gamma^{-1} \Im M & \Re M
\end{array}\right]\right)
$$

\subsection{Decentralized Fixed Modes}

The following definition of a decentralized fixed mode is made in Wang and Davison (1973).

Definition 4. (Decentralized Fixed Mode (DFM)). The LTI system (1) is said to have a DFM $\lambda \in \mathbb{C}$, if $\lambda \in \operatorname{sp}(A)$ and for all conformal $K_{i} \in \mathbb{R}^{m_{i} \times r_{i}}(i \in \underline{v})$ with the property that $(I-D K)^{-1}$ exists,

$$
\lambda \in \operatorname{sp}\left(A+B K(I-D K)^{-1} C\right)
$$

where $K:=\operatorname{block} \operatorname{diag}\left(K_{1}, \ldots, K_{v}\right)$.

An algebraic characterization of a DFM is given in Davison and Chang (1990).

Theorem 5. Given the LTI system $(1), \lambda \in \operatorname{sp}(A)$ is a DFM of (1) if and only if there exists a subset $\mathbf{P}=\left\{i_{1}, \ldots, i_{k}\right\}$ of $\underline{v}$ (i.e. $\mathbf{P} \subseteq \underline{v}$ ), and a complementary subset $\overline{\mathbf{P}}=$ $\left\{j_{1}, \ldots, j_{v-k}\right\}$ (i.e. $\left.\overline{\mathbf{P}}=\underline{v}-\mathbf{P}\right)$ such that

$$
\operatorname{rank}\left(\left[\begin{array}{cc}
A-\lambda I_{n} & B_{\overline{\mathbf{P}}} \\
C_{\mathbf{P}} & D_{\mathbf{P} \overline{\mathbf{P}}}
\end{array}\right]\right)<n
$$

where we denote $T(s, \mathbf{P}):=\left[\begin{array}{cc}A-s I & B_{\overline{\mathbf{P}}} \\ C_{\mathbf{P}} & D_{\mathbf{P} \overline{\mathbf{P}}}\end{array}\right]$ and is defined as follows

$1 \tau_{k}(M)$ defined in this paper is sometimes referred to as the real perturbation values of the second kind.

$$
\begin{aligned}
& {\left[\begin{array}{cc}
A-s I & B_{\overline{\mathbf{P}}} \\
C_{\mathbf{P}} & D_{\mathbf{P} \overline{\mathbf{P}}}
\end{array}\right]:=}
\end{aligned}
$$

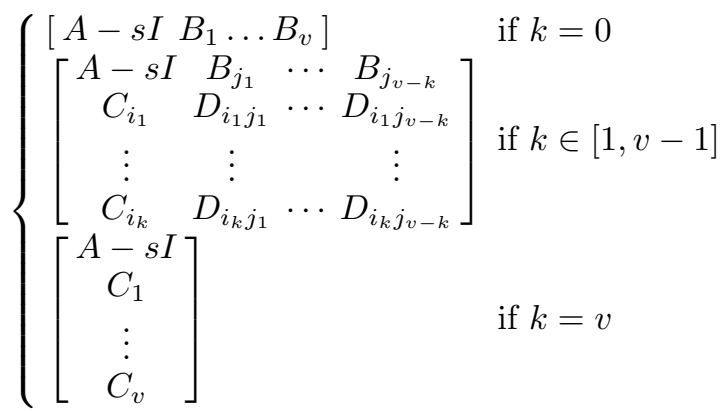

Furthermore, denote the dimensions of $B_{\overline{\mathbf{P}}}$ and $C_{\mathbf{P}}$ by $n \times m_{\overline{\mathbf{P}}}$ and $r_{\mathbf{P}} \times n$ respectively.

\subsection{Decentralized Fixed Mode Radius}

Following a similar approach as introduced in Vaz and Davison (1988), the DFM radius and unstable DFM radius are defined as follows.

Definition 6. (DFM Radius). Given a LTI system (1) that has no DFMs, the DFM radius of the system is defined as

$$
\begin{aligned}
& r_{\mathbb{F}}^{D F M}\left(\left[\begin{array}{c|c}
A \mid B \\
C
\end{array}\right]_{v}\right)=\inf \left\{\left\|\left[\begin{array}{cc}
\Delta_{A} & \Delta_{B} \\
\Delta_{C} & \Delta_{D}
\end{array}\right]\right\|\right. \\
& \Delta_{A} \in \mathbb{F}^{n \times n}, \Delta_{B} \in \mathbb{F}^{n \times m}, \Delta_{C} \in \mathbb{F}^{r \times n}, \Delta_{D} \in \mathbb{F}^{r \times m}, \\
& \left.\left[\begin{array}{c|c}
A+\Delta_{A} & B+\Delta_{B}
\end{array}\right]_{v} \text { has at least one DFM }\right\}
\end{aligned}
$$$$
\text { where } m=\sum_{i=1}^{v} m_{i}, r=\sum_{i=1}^{v} r_{i} \text {, and } \mathbb{F} \in\{\mathbb{R}, \mathbb{C}\} .{ }^{2}
$$

Definition 7. (Unstable DFM Radius). Likewise, given a LTI system (1) that has no unstable DFMs, the unstable DFM radius is defined as

$$
\begin{aligned}
& r_{\mathbb{F}}^{D F M_{+}}\left(\left[\frac{A \mid B}{C \mid D}\right]_{v}\right)=\inf \left\{\left\|\left[\begin{array}{cc}
\Delta_{A} & \Delta_{B} \\
\Delta_{C} & \Delta_{D}
\end{array}\right]\right\|\right. \\
& \Delta_{A} \in \mathbb{F}^{n \times n}, \Delta_{B} \in \mathbb{F}^{n \times m}, \Delta_{C} \in \mathbb{F}^{r \times n}, \Delta_{D} \in \mathbb{F}^{r \times m}, \\
& \left.\left[\begin{array}{c|c}
A+\Delta_{A} & B+\Delta_{B}
\end{array}\right]_{v} \text { has at least one unstable DFM }\right\}
\end{aligned}
$$

In Vaz and Davison (1988), it was shown that the complex DFM radius and the complex unstable DFM radius are respectively given by:

$$
r_{\mathbb{C}}^{D F M}\left(\left[\begin{array}{c|c}
A & B \\
\hline C & D
\end{array}\right]_{v}\right)=\min _{s \in \mathbb{C}} \min _{\mathbf{P} \subseteq \underline{v}} \sigma_{n}(T(s, \mathbf{P}))
$$

and

$$
r_{\mathbb{C}}^{D F M_{+}}\left(\left[\begin{array}{l|l}
A & B \\
\hline C & D
\end{array}\right]_{v}\right)=\min _{s \in \mathbb{C}_{+}} \min _{\mathbf{P} \subseteq \underline{v}} \sigma_{n}(T(s, \mathbf{P}))
$$

In Lam and Davison (2007), the real DFM radius and the real unstable DFM radius are respectively obtained as:

$$
r_{\mathbb{R}}^{D F M}\left(\left[\begin{array}{c|c}
A & B \\
\hline C & D
\end{array}\right]_{v}\right)=\min _{s \in \mathbb{C}} \min _{\mathbf{P} \subseteq \underline{v}} \tau_{n}(T(s, \mathbf{P}))
$$

and

$$
r_{\mathbb{R}}^{D F M_{+}}\left(\left[\begin{array}{c|c}
A & B \\
\hline C & D
\end{array}\right]_{v}\right)=\min _{s \in \mathbb{C}_{+}} \min _{\mathbf{P} \subseteq \underline{v}} \tau_{n}(T(s, \mathbf{P}))
$$

$2 r_{\mathbb{C}}^{D F M}$ (i.e. $\mathbb{F}=\mathbb{C}$ ) is called the complex DFM radius, whereas $r_{\mathbb{R}}^{D F M}$ is called the real DFM radius. 
We will make one further definition.

Definition 8. Given a LTI system (1) that has no DFM and given $s \in \mathbb{C}$, the modal DFM radius of (1) with respect to $s$, denoted by $r_{\mathbb{F}}^{s}$, is defined as

$$
r_{\mathbb{F}}^{s}\left(\left[\begin{array}{c|c}
A & B \\
\hline C & D
\end{array}\right]_{v}\right)=\inf \left\{\left\|\left[\begin{array}{cc}
\Delta_{A} & \Delta_{B} \\
\Delta_{C} & \Delta_{D}
\end{array}\right]\right\| \|\right.
$$

$\Delta_{A} \in \mathbb{F}^{n \times n}, \Delta_{B} \in \mathbb{F}^{n \times m}, \Delta_{C} \in \mathbb{F}^{r \times n}, \Delta_{D} \in \mathbb{F}^{r \times m}$,

$$
\left.\left[\begin{array}{c|c}
A+\Delta_{A} & B+\Delta_{B} \\
\hline C+\Delta_{C} & D+\Delta_{D}
\end{array}\right]_{v} \text { has at least one DFM at } s\right\}
$$

where $\mathbb{F} \in\{\mathbb{C}, \mathbb{R}\}$. The model complex and real DFM radius are respectively obtained as:

$$
r_{\mathbb{C}}^{s}\left(\left[\begin{array}{l|l}
A & B \\
\hline C & D
\end{array}\right]_{v}\right)=\min _{\mathbf{P} \subseteq \underline{v}} \sigma_{n}(T(s, \mathbf{P}))
$$

and

$$
r_{\mathbb{R}}^{s}\left(\left[\begin{array}{l|l}
A & B \\
\hline C & D
\end{array}\right]_{v}\right)=\min _{\mathbf{P} \subseteq \underline{v}} \tau_{n}(T(s, \mathbf{P}))
$$

\section{MAIN RESULTS}

The main focus of this paper is: i) to present an algorithm for computing the minimum norm perturbation that achieves the real DFM radius; ii) to discuss some properties of the real DFM radius; iii) to extend the real DFM radius to deal with structured perturbations; and iv) to extend the real DFM radius to deal with general decentralized information flow constraints.

\subsection{Constructing the perturbations that achieve $r_{\mathbb{R}}^{D F M}$}

Assume that the real DFM radius of $\left[\frac{A \mid B}{C \mid D}\right]_{v}$ is found to be $r_{\mathbb{R}}^{D F M}$ and is achieved at $s, \mathbf{P}$ and $\overline{\mathbf{P}}$. This section now presents a method for constructing the real perturbation matrix, $\Delta:=\left[\begin{array}{cc}\Delta_{A} & \Delta_{B} \\ \Delta_{C} & \Delta_{D}\end{array}\right]$, that achieves $r_{\mathbb{R}}^{D F M}$ (i.e. so that $\left.\left\|\left[\begin{array}{ll}\Delta_{A} & \Delta_{B} \\ \Delta_{C} & \Delta_{D}\end{array}\right]\right\|=r_{\mathbb{R}}^{D F M}\right)$.

Note that $\Delta$ can easily be derived from any matrix $\Delta_{s}:=$ $\left[\begin{array}{cc}\Delta_{A} & \Delta_{B_{\overline{\mathbf{P}}}} \\ \Delta_{C_{\mathbf{P}}} & \Delta_{D_{\mathbf{P} \overline{\mathbf{P}}}}\end{array}\right]$ that satisfies

$$
\operatorname{rank}\left(T(s, \mathbf{P})+\Delta_{s}\right)<n
$$

In particular, the rows/columns of $\Delta_{B}, \Delta_{C}$, and $\Delta_{D}$ corresponding to $\mathbf{P}$ and $\overline{\mathbf{P}}$ as defined by (6) can respectively be set to be equal to $\Delta_{B_{\overline{\mathbf{P}}}}, \Delta_{C_{\mathbf{P}}}$, and $\Delta_{D_{\mathbf{P} \overline{\mathbf{P}}}}$, while the remaining elements are set to zero. Hence, the main effort in constructing $\Delta$ is in finding $\Delta_{s}$. The following theorem found in Karow (2003) can be used to construct $\Delta_{s}$.

Theorem 9. Given $M \in \mathbb{C}^{q \times l}$ and $k \in \mathbb{N}$. If $\tau_{k}(M)=\infty$, then there exists no $\Delta \in \mathbb{R}^{q \times l}$ such that $\operatorname{rank}(M+\Delta)<k$. Suppose $\tau_{k}(M)<\infty$. Let $\mathcal{X}$ be any $(l-k+1)$-dimensional subspace of $\mathbb{C}^{l}$ satisfying the hermitian-symmetric inequality

$$
x^{*}\left(\tau_{k}(M)^{2} I_{l}-M^{*} M\right) x \geq\left|x^{T}\left(\tau_{k}(M)^{2} I_{l}-M^{T} M\right) x\right|
$$

for all $x \in \mathcal{X}$, and let $X \in \mathbb{C}^{l \times(l-k+1)}$ be any matrix whose columns form a basis of $\mathcal{X}$. Set

$$
\Delta:=-[\Re(M X) \Im(M X)][\Re(X) \Im(X)]^{+} \in \mathbb{R}^{q \times l}
$$

Then

$$
\operatorname{rank}(M+\Delta)<k \text { and } \quad\|\Delta\|=\tau_{k}(M)
$$

Hence from Theorem 9 , we immediately see that $\Delta_{s}$ can be constructed as follows:

$$
\Delta_{s}:=-\left[\Re\left(T(s, \mathbf{P}) X_{s}\right) \Im\left(T(s, \mathbf{P}) X_{s}\right)\right]\left[\Re\left(X_{s}\right) \Im\left(X_{s}\right)\right]^{+}
$$

where $X_{s}$ is a complex matrix whose columns form the basis for a subspace that satisfies

$$
x^{*} \hat{H} x \geq\left|x^{T} \hat{S} x\right|
$$

where $\hat{H}:=\left(r_{\mathbb{R}}^{D F M}\right)^{2} I-T(s, \mathbf{P})^{*} T(s, \mathbf{P})$ and $\hat{S}:=$ $\left(r_{\mathbb{R}}^{D F M}\right)^{2} I-T(s, \mathbf{P})^{T} T(s, \mathbf{P})$. Such a basis (i.e. $\left.X_{s}\right)$ can be obtained by performing a simultaneous block diagonalization of $\hat{H}$ and $\hat{S}$. We will consider two cases: i) when $s$ is real; and ii) when $s$ is complex.

Case (i): $s$ is real

When $s$ is real, $\hat{H}=\hat{S}$ are real symmetric. Hence, $(\hat{H}, \hat{S})$ can both be simultaneously diagonalized via a real orthogonal matrix $P$; i.e. $P^{T} \hat{H} P=P^{T} \hat{S} P=\Lambda$, where $\Lambda$ is a real diagonal matrix. Such a $P$ can be obtained by the Takagi decomposition of $\hat{H}{ }^{3}$ Finally, set $X_{s}=$ $\left[x_{1}, \ldots, x_{k}\right]$, where $x_{1}, \ldots, x_{k}$ are the columns of $P$ that satisfy $(20)$ and $k=m_{\overline{\mathbf{P}}}+1$.

Case (ii): $s$ is complex

Let $\mathcal{H} \mathcal{S}_{n}:=\left\{(H, S) \in \mathbb{C}^{n \times n} \times \mathbb{C}^{n \times n} \mid H^{*}=H, S^{T}=S\right\}$ be the set of $n \times n$ hermitian-symmetric matrix pairs. Furthermore, denote

$$
\begin{aligned}
\mathcal{H S}_{n}^{+}:= & \left\{(H, S) \in \mathcal{H S}_{n} \mid \operatorname{det}(H) \neq 0,\right. \\
& \left.C \text { is condiagonalizable }{ }^{4}, \text { where } C:=S^{-1} \bar{H}\right\}
\end{aligned}
$$

It is shown in Karow (2003) that the set $\mathcal{H S}_{n}^{+}$is an open and dense subset of $\mathcal{H} \mathcal{S}_{n}$. Also, the following is true.

Theorem 10. (Karow (2003)). Let $(H, S) \in \mathcal{H S}_{n}^{+}$and $C=$ $S^{-1} \bar{H}$. Then $C \bar{C}$ has non-negative real eigenvalues, the nonreal eigenvalues of $C \bar{C}$ occur in complex conjugate pairs, and there exists an invertible matrix $P \in \mathbb{C}^{n \times n}$ such that

$$
\begin{aligned}
P^{*} H P= & \text { block } \operatorname{diag}\left\{\lambda_{1}, \ldots, \lambda_{\rho}, \lambda_{\rho+1}, \ldots, \lambda_{\rho+l},\right. \\
& \left.\lambda_{\rho+l+1}, \ldots, \lambda_{r},\left[\begin{array}{cc}
0 & \overline{\nu_{1}} \\
\nu_{1} & 0
\end{array}\right], \ldots,\left[\begin{array}{cc}
0 & \overline{\nu_{s}} \\
\nu_{s} & 0
\end{array}\right]\right\} \\
P^{T} S P= & I_{n}
\end{aligned}
$$

where

- $\lambda_{i} \in \mathbb{R}$ and $\lambda_{i}^{2}$ is a real eigenvalue of $C \bar{C}$, for $i=$ $1, \ldots, r$

3 Since $\hat{H}$ and $\hat{S}$ are real symmetric, the real Schur decomposition can also be used.

${ }^{4}$ i.e. there exists a nonsingular $R \in \mathbb{C}^{n \times n}$ such that $R^{-1} C \bar{R}$ is diagonal. 
- $\Im \nu_{i} \in \mathbb{C} \backslash \mathbb{R}$ with $\Im \nu_{i}>0$ and $\nu_{i}^{2},{\overline{\nu_{i}}}^{2}$ are the nonreal eigenvalues of $C \bar{C}$, for $i=1, \ldots, s$

- $1>\lambda_{1} \geq \cdots \geq \lambda_{\rho} \geq-1$

- $\lambda_{i} \geq 1$ for $\rho+1 \leq i \leq \rho+l$

- $\lambda_{i}<-1$ for $\rho+l+1 \leq i \leq r$

Furthermore, the columns of $P$ form a basis of eigenvectors for $C \bar{C}$.

Let $(\hat{H}, \hat{S}) \in \mathcal{H S}_{n+m_{\overline{\mathbf{P}}}}^{+}$, where $\hat{H}$ and $\hat{S}$ are given in (20), then by Theorem 10 , there exists a $P$ that can simultaneously block diagonalize $\hat{H}$ and $\hat{S}$ into the form given by (21). Such a $P$ can be constructed by a procedure in Karow (2003) and is summarized below.

First, let $\mu_{1}, \ldots, \mu_{r}$ be the real eigenvalues of $C \bar{C}$, where $C=\hat{S}^{-1} \hat{H}$, and $\mu_{r+1}, \ldots, \mu_{r+s}$ be the eigenvalues of $C \bar{C}$ with positive imaginary part. Now, let $u_{1}, \ldots, u_{r}$ be the eigenvectors corresponding to $\mu_{1}, \ldots, \mu_{r}$, and $v_{1}, \ldots, v_{s}$ be the eigenvectors corresponding to $\mu_{r+1}, \ldots, \mu_{r+s}$. (Note: $C \overline{v_{1}}, \ldots, C \overline{v_{s}}$ are eigenvectors corresponding to $\left.\overline{\mu_{r+1}}, \ldots, \overline{\mu_{r+s}}.\right)$ Define

$$
Q=\left[u_{1}, \ldots, u_{r}, v_{1}, C \overline{v_{1}}, \ldots, v_{s}, C \overline{v_{s}}\right]
$$

and let $P$ be as follows:

$$
P=Q \operatorname{diag}\left(\alpha_{1}^{-1}, \ldots, \alpha_{r}^{-1}, \beta_{1}^{-1}, \overline{\gamma_{1}^{-1} \beta_{1}^{-1}}, \ldots, \beta_{s}^{-1}, \overline{\gamma_{s}^{-1} \beta_{s}^{-1}}\right)
$$

where $\alpha_{j}, \beta_{j}, \gamma_{j} \in \mathbb{C} \backslash\{0\}$ satisfy

$$
\begin{array}{ll}
\alpha_{j}^{2}=u_{j}^{T} \hat{S} u_{j} & 1 \leq j \leq r, \\
\beta_{j}^{2}=v_{j}^{T} \hat{S} v_{j} & 1 \leq j \leq s, \\
\gamma_{j}^{2}=\mu_{r+j} & 1 \leq j \leq s
\end{array}
$$

Such a $P:=\left[p_{1}, \ldots, p_{r+2 s}\right]$ will simultaneously block diagonalize $\hat{H}$ and $\hat{S}$ in the form given by (21). ${ }^{5}$ Then,

- for each $\lambda_{j} \geq 1$, set $a_{j}:=p_{\rho+j}$, for $j=1, \ldots, l$

- for each complex pair $\left(\nu_{j}, \overline{\nu_{j}}\right)$, set $b_{j}:=p_{r+2 j-1}+$ $i p_{r+2 j}$, for $j=1, \ldots, s$

- for the set of eigenvalues $\left\{\lambda_{1}, \ldots, \lambda_{\rho}\right\}$, there exists $w=m_{\overline{\mathbf{P}}}-l-s+1$ pairs such that $\lambda_{j}+\lambda_{2 w+1-j} \geq 0$; set $c_{j}:=p_{j}+i p_{2 w+1-j}$ for $j=1, \ldots, w$.

Finally, set $X_{s}=\left[x_{1}, \ldots, x_{m_{\overline{\mathrm{P}}}+1}\right]$, where $x_{1}, \ldots, x_{m_{\overline{\mathrm{P}}}+1}$ are now the columns of $\left[a_{1}, \ldots, a_{l}, b_{1}, \ldots, b_{s}, c_{1}, \ldots, c_{w}\right]$ that satisfy (20), and substitute $X_{s}$ into (19) to obtain $\Delta_{s}$.

\subsection{Properties of the real DFM radius}

Lemma 11. The real DFM radius of (1) is invariant under real orthogonal coordinate transformations. In particular, let $z=Q x$ where $Q$ is real orthogonal and consider the system:

$$
\begin{aligned}
\dot{z} & =\tilde{A} z+\sum_{i=1}^{v} \tilde{B}_{i} u_{i} \\
y_{i} & =\tilde{C}_{i} z+\sum_{j=1}^{v} \tilde{D}_{i j} u_{j} \quad(i \in \underline{v})
\end{aligned}
$$

\footnotetext{
5 To achieve the particular ordering of $\lambda_{1}, \ldots, \lambda_{r}$ as specified in (21), the first $r$ columns of $P$ may need to be rearranged in a different order.
}

where $\tilde{A}:=Q A Q^{T}, \tilde{B}_{i}:=Q B_{i}, \tilde{C}_{i}:=C_{i} Q^{T}$ and $\tilde{D}_{i j}:=$ $D_{i j}$, for $i, j \in \underline{v}$. Then

$$
r_{\mathbb{R}}^{D F M}\left(\left[\begin{array}{c|c}
\tilde{A} & \tilde{B} \\
\hline \tilde{C} & \tilde{D}
\end{array}\right]_{v}\right)=r_{\mathbb{R}}^{D F M}\left(\left[\begin{array}{l|l}
A & B \\
\hline C & D
\end{array}\right]_{v}\right)
$$

where $\tilde{B}, \tilde{C}$, and $\tilde{D}$ are defined in a similar way as matrices $B, C$, and $D$ respectively.

\section{Proof.}

$$
\begin{aligned}
r_{\mathbb{R}}^{D F M} & \left(\left[\begin{array}{c|c}
\tilde{A} & \tilde{B} \\
\hline \tilde{C} & \tilde{D}
\end{array}\right]_{v}\right) \\
& =\min _{s \in \mathbb{C}} \min _{\mathbf{P} \subseteq \underline{v}} \tau_{n}\left(\left[\begin{array}{cc}
Q & 0 \\
0 & I
\end{array}\right] T(s, \mathbf{P})\left[\begin{array}{cc}
Q^{T} & 0 \\
0 & I
\end{array}\right]\right) \\
& =\min _{s \in \mathbb{C}} \min _{\mathbf{P} \subseteq \underline{v}} \tau_{n}(T(s, \mathbf{P})) \quad \text { by Lemma } 2 \text { (iii) }
\end{aligned}
$$

Lemma 12. Given $s \in \mathbb{C}$, then

$$
r_{\mathbb{R}}^{s}\left(\left[\begin{array}{l|l}
A & B \\
\hline C & D
\end{array}\right]_{v}\right)=r_{\mathbb{R}}^{\bar{s}}\left(\left[\begin{array}{l|l}
A & B \\
\hline C & D
\end{array}\right]_{v}\right)
$$

\section{Proof.}

$$
\begin{aligned}
r_{\mathbb{R}}^{s}\left(\left[\frac{A \mid B}{C} \mid D\right]_{v}\right) & =\min _{\mathbf{P} \subseteq \underline{v}} \tau_{n}(T(s, \mathbf{P})) \\
& =\min _{\mathbf{P} \subseteq \underline{v}} \tau_{n}(T(\bar{s}, \mathbf{P})) \text { by Lemma } 2 \text { (ii) }
\end{aligned}
$$

Remark 13. An immediate result of Lemma 12 is that if a given $s$ achieves the real DFM radius, then $\bar{s}$ also achieves it. More importantly, $r_{\mathbb{R}}^{s}\left(\left[\begin{array}{l|l}A \mid B \\ \hline C \mid D\end{array}\right]_{v}\right)$ is therefore a mirrored image with respect to the real axis. Hence, the search for the global minimizer that achieves the real DFM radius can be restricted to either the closed upper or lower half of the complex plane.

\subsection{Real DFM radius with structured perturbations}

Definition 14. Given an LTI system (1) that has no DFMs. Consider the following structured perturbations:

$$
\begin{aligned}
A & \rightarrow A+E_{1} \Delta_{A} F_{1} \\
B_{i} & \rightarrow B_{i}+E_{1} \Delta_{B_{i}} f_{2 i} \quad i \in \underline{v} \\
C_{i} & \rightarrow C_{i}+e_{2 i} \Delta_{C_{i}} F_{1} \quad i \in \underline{v} \\
D_{i j} & \rightarrow D_{i j}+e_{2 i} \Delta_{D_{i j}} f_{2 j} \quad i, j \in \underline{v}
\end{aligned}
$$

where $E_{1}, F_{1}, E_{2}:=\operatorname{block} \operatorname{diag}\left(e_{21}, \ldots, e_{2 v}\right)$, and $F_{2}:=$ block diag $\left(f_{21}, \ldots, f_{2 v}\right)$ are all nonsingular. The structured real DFM radius of the system is defined as

$r_{\mathbb{R}, \text { struct }}^{D F M}\left(\left[\frac{A \mid B}{C} \mid D\right]_{v}\right)=\inf \left\{\left\|\left[\begin{array}{cc}\Delta_{A} & \Delta_{B} \\ \Delta_{C} & \Delta_{D}\end{array}\right]\right\| \|\right.$

$\Delta_{A} \in \mathbb{R}^{n \times n}, \Delta_{B} \in \mathbb{R}^{n \times m}, \Delta_{C} \in \mathbb{R}^{r \times n}, \Delta_{D} \in \mathbb{R}^{r \times m}$,

$\left[\begin{array}{c|c}A+E_{1} \Delta_{A} F_{1} & B+E_{1} \Delta_{B} F_{2} \\ \hline C+E_{2} \Delta_{C} F_{1} & D+E_{2} \Delta_{D} F_{2}\end{array}\right]_{v}$ has at least one DFM $\}$

Lemma 15.

$$
\begin{aligned}
& r_{\mathbb{R}, \text { struct }}^{D F M}\left(\left[\frac{A \mid B}{C \mid D}\right]_{v}\right) \\
& \quad=\min _{s \in \mathbb{C}} \min _{\mathbf{P} \subseteq \underline{v}} \tau_{n}\left(\left[\begin{array}{cc}
E_{1} & 0 \\
0 & E_{2 \mathbf{P}}
\end{array}\right]^{-1} T(s, \mathbf{P})\left[\begin{array}{cc}
F_{1} & 0 \\
0 & F_{2 \overline{\mathbf{P}}}
\end{array}\right]^{-1}\right)
\end{aligned}
$$


where for the partition $\mathbf{P}:=\left\{i_{1}, \ldots, i_{k}\right\}$, and $\overline{\mathbf{P}}:=$ $\left\{j_{1}, \ldots, j_{v-k}\right\}$, we denote $E_{2 \mathbf{P}}:=\operatorname{block} \operatorname{diag}\left(e_{2 i_{1}}, \ldots, e_{2 i_{k}}\right)$ and $F_{2 \overline{\mathbf{P}}}:=$ block $\operatorname{diag}\left(f_{2 j_{1}}, \ldots, f_{2 j_{v-k}}\right)$.

Proof. The proof directly follows given that for nonsingular $E$ and $F$,

$$
\operatorname{rank}(M+E \Delta F)=\operatorname{rank}\left(E^{-1} M F^{-1}+\Delta\right)
$$

\subsection{General information flow constraints}

In Lam and Davison (2007), the real DFM radius (11) is defined for the decentralized system (1) that is subject to a diagonal information flow constraint, $K$; i.e.

$$
\begin{gathered}
u=K y \\
\text { where } u=\left[u_{1}^{T}, \cdots, u_{v}^{T}\right]^{T}, y=\left[y_{1}^{T}, \cdots, y_{v}^{T}\right]^{T}, \text { and } \\
K \in\left\{K \in \mathbb{R}^{m \times r} \mid K=\operatorname{block} \operatorname{diag}\left(K_{1}, K_{2}, \ldots, K_{v}\right),\right. \\
\left.K_{i} \in \mathbb{R}^{m_{i} \times r_{i}}, i=1, \ldots, v, \operatorname{det}(I-D K) \neq 0\right\}
\end{gathered}
$$

In this section, the real DFM radius is generalized to general information flow constraints, i.e.

$$
K \in\left\{K \in \mathbb{R}^{m \times r} \mid K=\left[\begin{array}{ccc}
K_{11} & \cdots & K_{1 v} \\
\vdots & & \vdots \\
K_{v 1} & \cdots & K_{v v}
\end{array}\right], K_{i j} \in \mathbb{R}^{m_{i} \times r_{j}}\right.
$$$$
i, j=1, \ldots, v\}
$$

where any of the elements of $K$ can be constrained to be zero, for systems with $D=0$.

Lemma 16. The decentralized LTI system (1) with $D=0$ and a general information flow constraint (32), $K$, can be written as an alternative decentralized system

$$
\begin{gathered}
\dot{x}=A x+\sum_{i=1}^{\tilde{v}} \tilde{B}_{i} \tilde{u}_{i} \\
\tilde{y}_{i}=\tilde{C}_{i} x \quad(i \in \underline{\tilde{v}})
\end{gathered}
$$

subject to a block diagonal information flow constraint $(31), \tilde{K}$; i.e.

$$
\begin{gathered}
\tilde{u}=\tilde{K} \tilde{y} \\
\text { where } \tilde{u}=\left[\tilde{u}_{1}^{T}, \cdots, \tilde{u}_{\tilde{v}}^{T}\right]^{T} \text { and } \tilde{y}=\left[\tilde{y}_{1}^{T}, \cdots, \tilde{y}_{\tilde{v}}^{T}\right]^{T} .
\end{gathered}
$$

Proof. For $i \in \underline{v}$, let $k_{i}$ denote the number of non-zero block matrices in the (ordered) set $K_{i}:=\left\{K_{i 1}, \ldots, K_{i v}\right\}$, where $K_{i j}$ are defined in (32) for $j=1, \ldots, v$, and let $h_{i, j} \in\left\{h_{i, 1}, \ldots, h_{i, k_{i}}\right\}$ be an index where $K_{i\left(h_{i, j}\right)}$ is the $j$-th non-zero block matrix of $K_{i}$, for $j=1, \ldots, k_{i}$. Now for $i \in \underline{v}$, let

$$
\begin{array}{ll}
\tilde{B}_{k_{1}+\cdots+k_{i-1}+j}=B_{i} & \text { for } j=1, \ldots, k_{i} \\
\tilde{C}_{k_{1}+\cdots+k_{i-1}+j}=C_{h_{i, j}} & \text { for } j=1, \ldots, k_{i}
\end{array}
$$

It can easily be verified that the original system (1) with the general information flow $K$ is equivalent to the alternative system (33) (where $\tilde{v}=\sum_{i=1}^{v} k_{i}$, and $\tilde{B}_{i}$ and $\tilde{C}_{i}$ are constructed as above, for $i \in \tilde{v}$ ) with the diagonal information flow constraint:

$$
\begin{array}{r}
\tilde{K}=\text { block } \operatorname{diag}\left(K_{1, h_{1,1}}, \ldots, K_{1, h_{1, k_{1}}}, K_{2, h_{2,1}}, \ldots, K_{2, h_{2, k_{2}}},\right. \\
\left.\ldots, K_{v, h_{v, 1}}, \ldots, K_{v, h_{v, k_{v}}}\right)
\end{array}
$$

Hence by Lemma 16, one can compute the real DFM radius of a decentralized system with a general information flow constraint by first reformulating the system such that a diagonal information flow constraint is used. The previous results (e.g. (11) and (12)) can then be applied to the new system after removing repeated columns and rows in $T(s, \mathbf{P})$ that arise from applying Lemma 16. To illustrate the latter point, it is best to use an example.

Example 17. Consider the following system with two control stations and subject to the general information flow constraint $K=\left[\begin{array}{cc}K_{11} & K_{12} \\ 0 & K_{22}\end{array}\right]$ :

$$
\begin{aligned}
\dot{x} & =A x+B_{1} u_{1}+B_{2} u_{2} \\
y_{1} & =C_{1} x, \quad y_{2}=C_{2} x
\end{aligned}
$$

The system (34) can be rewritten as

$$
\begin{aligned}
\dot{x} & =A x+B_{1} \tilde{u}_{1}+B_{1} \tilde{u}_{2}+B_{2} \tilde{u}_{3} \\
\tilde{y}_{1} & =C_{1} x, \quad \tilde{y}_{2}=C_{2} x, \quad \tilde{y}_{3}=C_{2} x
\end{aligned}
$$

where the information flow constraint is given by $\tilde{K}=$ block diag $\left(K_{11}, K_{12}, K_{22}\right)$. Given $s \in \mathbb{C}$, a direct application of (13) to compute the modal real DFM radius of system (35) with respect to $s$ requires the computation of the $n$-th real perturbation values of the following matrices: $\left[\begin{array}{llll}A-s I & B_{1} & B_{1} & B_{2}\end{array}\right],\left[\begin{array}{cc}A-s I & B_{1} \\ C_{1} & 0 \\ C_{2} & 0\end{array}\right],\left[\begin{array}{cc}A-s I & B_{1} \\ C_{2} & 0 \\ C_{2} & 0\end{array}\right]$, etc.

However, note that $B_{1}$ appears twice in the first matrix. Therefore to avoid perturbing each $B_{1}$ 's independently, the $n$-th real perturbation value of [ $\left.A-s I B_{1} B_{2}\right]$ should be computed instead. Likewise, all of the other matrices (i.e. $T(s, \mathbf{P}))$ that have repeated columns and/or rows obtained from applying Lemma 16 (e.g. the third matrix with repeated $C_{2}$ ) should have the repeated columns and rows removed before applying any of the previous results.

\section{NUMERICAL EXAMPLE}

Consider the following system, which has eigenvalues $\{-0.6956,-1.358 \pm 1.029 i\}$ and has two control stations, subject to the information flow constraint $K=\left[\begin{array}{cc}\times & 0 \\ 0 & \times\end{array}\right]$ :

$$
\begin{aligned}
\dot{x} & =\left[\begin{array}{ccc}
0 & -1 & -1 \\
1 & 1 & 1 \\
2 & 3 & 1
\end{array}\right] x+\left[\begin{array}{l}
1 \\
0 \\
0
\end{array}\right] u_{1}+\left[\begin{array}{c}
0 \\
0.1 \\
0
\end{array}\right] u_{2} \\
y_{1} & =\left[\begin{array}{lll}
0 & 0.01 & 0
\end{array}\right] x, \quad y_{2}=\left[\begin{array}{lll}
1 & 0 & 0.01
\end{array}\right] x
\end{aligned}
$$

By (11), the real DFM radius is found to be $7.902_{10^{-2}}$, which is achieved at $s=1.336 \pm 1.034 i, \mathbf{P}=\{1\}$, and $\overline{\mathbf{P}}=\{2\}$. Using the procedure presented in Section 3.1, the corresponding system perturbation is computed to be:

$$
\begin{aligned}
& \Delta:=\left[\begin{array}{c|c|c}
\Delta_{A} & \Delta_{b_{1}} & \Delta_{b_{2}} \\
\hline \Delta_{c_{1}} & \Delta_{d_{11}} & \Delta_{d_{12}} \\
\hline \Delta_{c_{2}} & \Delta_{d_{21}} & \Delta_{d_{22}}
\end{array}\right]= \\
& {\left[\begin{array}{ccc|c|c}
-2.14_{10^{-2}} & 4.45_{10^{-2}} & -5.42_{10^{-2}} & 0 & -1.78_{10^{-2}} \\
0 & 0 & 0 & 0 & -6.24_{10^{-2}} \\
8.45_{10^{-3}} & -1.76_{10^{-2}} & 2.14_{10^{-2}} & 0 & -4.50_{10^{-2}} \\
\hline 0 & -1.00_{10^{-2}} & 0 & 0 & 0 \\
\hline 0 & 0 & 0 & 0 & 0
\end{array}\right]}
\end{aligned}
$$


It can easily be verified that the norm of the perturbation (37) is equal to the real DFM radius $7.902_{10^{-2}}$ and that the perturbed system has a DFM at $s=1.336 \pm 1.034 i$.

Now consider the same system (36) with the information flow constraints $K=\left[\begin{array}{cc}\times & \times \\ 0 & \times\end{array}\right]$. Using the method presented in Section 3.4, the real DFM radius is found to be 0.1107 , which is achieved at $s=-0.6981, \mathbf{P}=\{\}$ and $\overline{\mathbf{P}}=\{1,2\}$. The following perturbation achieves the real DFM radius of 0.1107 :

\section{$\Delta=$}

$\left[\begin{array}{ccc|c|c}1.88_{10^{-3}} & -6.19_{10^{-4}} & -9.31_{10^{-4}} & -2.89_{10^{-3}} & -4.72_{10^{-3}} \\ 3.06_{10^{-2}} & -1.01_{10^{-2}} & -1.52_{10^{-2}} & -4.72_{10^{-2}} & -7.71_{10^{-2}} \\ -1.66_{10^{-2}} & 5.47_{10^{-3}} & 8.23_{10^{-3}} & 2.56_{10^{-2}} & 4.17_{10^{-2}} \\ \hline 0 & 0 & 0 & 0 & 0 \\ \hline 0 & 0 & 0 & 0 & 0\end{array}\right]$

where the perturbed system has a DFM at $s=-0.6981$ with respect to the information flow constraint $K=$ $\left[\begin{array}{cc}\times & \times \\ 0 & \times\end{array}\right]$.

\section{APPLICATION OF THE REAL DFM RADIUS}

Given a multi-input multi-output system that is to be controlled by a decentralized LTI controller, the real DFM radius can be used to determine how the inputs and outputs should be paired in order to obtain a robust decentralized control system structure with respect to parametric perturbations. In particular, one can compute the real DFM radius of the system with various input/output pairing combinations, and then chose the pairing that maximizes the real DFM radius. As a preliminary study, this section will compare the pairing decisions obtained using the real DFM radius with using the so-called Relative Gain Array (RGA) approach (see Bristol (1996)), which is an approach widely used in industry.

Given a stable system $(C, A, B, D)$, the RGA is defined as:

$$
\Gamma_{R G A}=G(0) \otimes\left(G(0)^{T}\right)^{-1}
$$

where $\otimes$ denotes element-by-element product, and $G(s):=$ $C(s I-A)^{-1} B+D$. In this case the RGA uses only the steady-state properties of the input/output behaviour of a given system. The following example shows how the real DFM radius can be used to choose the "optimal" input/output pairing, and shows, in contrast, that the RGA can lead to erroneous results.

\subsection{Counterexample to $R G A$ approach}

Given the stable system:

$$
\begin{aligned}
\dot{x} & =\left[\begin{array}{ccc}
-1 & 0 & 0 \\
0 & -0.01 & 0 \\
0 & 0 & -3
\end{array}\right] x+\left[\begin{array}{ll}
1 & 0 \\
0 & 1 \\
0 & 1
\end{array}\right]\left[\begin{array}{l}
u_{1} \\
u_{2}
\end{array}\right] \\
{\left[\begin{array}{l}
y_{1} \\
y_{2}
\end{array}\right] } & =\left[\begin{array}{lll}
1 & 1 & 0 \\
0 & 0 & 1
\end{array}\right] x
\end{aligned}
$$

the RGA is obtained to be $\Gamma_{R G A}=\left[\begin{array}{ll}1 & 0 \\ 0 & 1\end{array}\right]$, which suggests that the following pairing should be used to control the system (40) (Bristol (1996)):

$$
\left[\begin{array}{l}
u_{1} \\
u_{2}
\end{array}\right]=\left[\begin{array}{ll}
\times & 0 \\
0 & \times
\end{array}\right]\left[\begin{array}{l}
y_{1} \\
y_{2}
\end{array}\right]
$$

However, the real DFM radius of (40) for the information flow constraint:

$$
\left[\begin{array}{l}
u_{1} \\
u_{2}
\end{array}\right]=\left[\begin{array}{ll}
0 & \times \\
\times & 0
\end{array}\right]\left[\begin{array}{l}
y_{1} \\
y_{2}
\end{array}\right]
$$

is 0.2333 corresponding to the mode -0.7668 , whereas the real DFM radius of (40) for the information flow constraint (41) is zero, corresponding to the mode -0.01! This implies that if the pairing (41), as recommended by the RGA method is used, then the resulting controller would be unable to control the dominant mode $(-0.01)$ of the plant! On the other hand, if the pairing (42), as suggested by the real DFM radius is used, then all modes of the system can easily be controlled.

\section{CONCLUSIONS}

Various new results and properties regarding the real DFM radius are presented in this paper, which includes the extension of the radius to deal with structured parametric perturbations and general information flow constraints. A procedure for computing the actual system perturbations, which give rise to the real DFM radius, is also presented. A study is also carried out using the real DFM radius to determine how to pair system inputs and outputs such that the resulting control system structure is robust. An example in this case is presented to show how effective the real DFM radius approach can be compared to the RGA method.

\section{REFERENCES}

B. Bernhardsson, A. Rantzer, and L. Qiu. A summary on the real stability radius and real perturbation values. In Hurwitz Centennial on Stability Theory, pages 11-18. Birkhäuser Verlag, 1995.

B. Bernhardsson, A. Rantzer, and L. Qiu. Real perturbation values and real quadratic forms in a complex vector space. Linear Algebra and its Applications, 270:131-154, 1998.

E. H. Bristol. On a new measure of interaction for multivariable process control. IEEE Trans. Automat. Contr., 11(1):133-134, 1996.

E. J. Davison and T. N. Chang. Decentralized stabilization and pole assignment for general proper systems. IEEE Trans. Automat. Contr., 35(6):652-664, June 1990.

R. Eising. Between controllable and uncontrollable. Systems \& Control Letters, 4:263-264, 1984.

D. Hinrichsen and A. J. Pritchard. Mathematical Systems Theory I: Modelling, State Space Analysis, Stability and Robustness. Springer-Verlag, Berlin, 2005.

M. Karow. Geometry of Spectral Value Sets. PhD thesis, University of Bremen, Germany, 2003.

S. Lam and E. J. Davison. The real decentralized fixed mode radius of LTI systems. In $46^{\text {th }}$ IEEE Conf. on Decision and Control, pages 3036-3041, 2007.

A. F. Vaz and E. J. Davison. A measure for the decentralized assignability of eigenvalues. Systems $\&$ Control Letters, 10:191-199, 1988.

S. H. Wang and E. J. Davison. On the stabilization of decentralized control systems. IEEE Trans. Automat. Contr., 18:473-478, 1973. 